\title{
Localized junctional epidermolysis bullosa, non-Herlitz type
}

INSERM

\section{Source}

INSERM. (1999). Orphanet: an online rare disease and orphan drug data base. Localized junctional epidermolysis bullosa, non-Herlitz type. ORPHA:251393

Junctional epidermolysis bullosa, localized non-Herlitz-type is a form of non-Herlitz junctional epidermolysis bullosa (JEB- $\mathrm{nH}$, see this term) characterized by localized blistering, and dystrophic or absent nails. 